\title{
LA CULTURA DE LA VIOLENCIA
}

\section{Milton Hernán Bentancor ${ }^{1}$}

Universidade de Caxias do Sul

\section{Resumen}

La actual literatura hispanoamericana recorre caminos que se hacen necesarios como expresión artística posterior al todopoderoso "Boom". En estos nuevos senderos aparecen nombres, obras y técnicas que ofrecen nuevas perspectivas para la labor creativa, tanto desde la temática como desde las voces que se escuchan. En este artículo deseamos analizar cómo la cultura latinoamericana se ha impregnado de violencia - en sus más diversas formas - y cómo esta se refleja en la creación literaria de diversos autores.

\section{Resumo}

A atual literatura hispano-americana percorre caminhos que se fazem necessários como expressão artística posterior ao todo-poderoso "Boom". Nesses novos caminhos aparecem nomes, obras e técnicas que oferecem novas perspectivas para o trabalho criativo, tanto no tocante à temática quanto no que diz respeito às vozes que se escutam. Neste artigo, desejamos analisar como a cultura latino-americana se tem impregnado de violência - nas suas mais diversas formas - e como essa violência se reflete na criação literária de diversos autores.

\section{Abstract}

The current American literature crosses paths that are necessary as artistic expression after the almighty "Boom". In these new paths names, works and techniques that offer new perspectives for creative work, both the subject and from the voices heard appear. In this article we want to analyze how Latin American culture has been steeped in violence -in its various forms- and how this is reflected in literary works of various authors.

\section{Palabras clave}

Literatura hispanoamericana. Siglo XXI. Nuevas voces narrativas. Cultura. Violencia.

\section{Palavras-chave}

Literatura hispano americana. Século XXI. Novas vozes narrativas. Cultura. Violência.

\section{Keywords}

Latin American literature. XXI Century. New narrative voices. Culture. Violence.

\footnotetext{
${ }^{1}$ El autor es Doctor en Letras. Noviembre de 2015.
} 


\section{Lo que era y lo que no es más}

Los autores que están escribiendo literatura en estos últimos años, los primeros del siglo XXI, se dividen -generacionalmente- entre los "hijos" y los "nietos" del Boom. No es una carga fácil de llevar, pero estos escritores -jóvenes, en comparación con Vargas Llosa, último representante vivo de aquella generación dorada- optaron por aprovechar la sombra ofrecida por los maestros y hablar de otra cosa.

Aquel compromiso -artístico y, en algunos casos, vital- que los autores de la década de los 60 tenían -con lo político y social- se perdió en la desilusión -con casi todo- de los jóvenes que entraron en la universidad en los últimos años del siglo pasado y en los primeros del presente. Esa decepción generalizada se traduce en textos que se alejan, cada vez más, de las dictaduras que asolaron nuestros países, de las torturas y las cárceles que avergüenzan nuestras historias y de las obligaciones sociales que muchos escritores de la segunda mitad del siglo XX sentían y exigían de los creadores para acercarse a la introspección o a la descripción sin compromiso social.

Como observa Gioconda Belli en el prólogo de la colección Retrato de poeta con joven errante, hablando específicamente de los jóvenes poetas nicaragüenses del siglo XXI, pero que se podría generalizar para los nuevos escritores hispanoamericanos:

\footnotetext{
El impulso de esta generación, que yo llamaría la Generación del Desasosiego, es el de salirse de ese entorno podrido donde su cabeza juvenil no encuentra ni reposo, ni propósito, ni guía, y emprender el viaje interior, ya sea hacia la desilusión o hacia la aparente fatalidad de la condición humana. ${ }^{1}$
}

Siendo así, la mítica Plaza de Mayo en Buenos Aires, deja de ser el símbolo de la lucha de las Madres y Abuelas de Plaza, para transformarse en un punto de referencia turístico o apenas un lugar de paso para algún personaje -casi- desprevenido. Managua deja de ser el espacio del enfrentamiento entre sandinistas y opositores para ganar aires de ciudad para vivir y tener hijos (o no). Sendero Luminoso se pierde en el tiempo como un mal recuerdo de bombas y terror.

Para intentar comprender mejor el concepto central de este artículo volvamos a las ideas aristotélicas $^{2}$ que sirven como base, incluso, para la definición que la Real Academia presenta en su diccionario cuando habla de violencia: "Acción que se desarrolla contra el natural modo de proceder"3. Con esta definición podemos inferir que -como acontece en los cuentos de los narradores que estudiaremos en este artículo- la violencia impone el desorden; nos animamos a dar un paso más y aseverar que en estas narraciones impone, de alguna manera, el caos.

Una segunda característica de la violencia es que atropella al otro; a quien la sufre, lo somete. Lo hace de diferentes maneras, con distintos matices, pero siempre marcando que el violento es quien manda, quien ordena, quien está en condiciones de exigir. El otro, el violentado, apenas conseguirá padecerla; en la mayoría de los casos, en silencio.

Esta violencia parece estar cimentada en un individualismo absoluto (ver SIMMEL ${ }^{4}$ y VELHO VI, $^{5}$ donde el diálogo se pierde y las acciones responden más a instintos (algunos más bárbaros que otros) que

\footnotetext{
${ }^{1}$ BELL, Gioconda (pról.). Retrato de poeta con joven errante: muestra de poesía nicaragüense escrita por jóvenes (20002005) Por Ruiz Udiel, Francisco y Juárez Polanco, Ulises. Managua: Ediciones Internacionales, 2005 , p. 15.

${ }^{2}$ ARISTÓTELES. Política. Tradução, introdução e notas de Mario da Gama Kury. 3. ed. Brasilia: Editora UNB, 1997.

${ }^{3}$ REAL ACADEMIA ESPAÑOLA. Diccionario de la lengua española. Disponible en: http://dle.rae.es/?id=brdBvt6. Web. 05 de noviembre de 2015.

${ }^{4}$ SIMMEL, Georg. On individuality and social forms. Org. Donald Levine. Chicago: University of Chicago Press, 1971.

${ }^{5}$ VELHO, Gilberto. Individualismo e cultura: notas para uma antropologia da sociedade contemporânea. Rio de Janeiro: Zahar, 1981.
} 
a valores o relaciones maduras o -al menos- socialmente aceptables; más allá de la hipotética convivencia que se da entre los habitantes de las metrópolis.

Como perspicazmente observa Alves de Carvalho ${ }^{6}$ en su estudio:

Ao se tomar como ponto de partida a perspectiva teórica sobre conflito social em Simmel, é possível compreender a violência contemporânea como um conceito muito mais amplo, que vai além das questões de socialização e relações recíprocas marcadas pelo conflito social.

Mesmo na possibilidade de ser confundida como o conflito social, a sociologia evidenciou que a violência, já instrumentalizada, provém de múltiplos fatores políticos, culturais, econômicos ou sociais, que demarcam sua complexidade quando esta se torna difusa, sem referências estáveis, sem âmbito e inexplicável. Porém, ela deixa brechas para se reconhecer que seu ponto de partida e chegada ocorre no campo simbólico dos sujeitos, quando agride a alma e rebaixa a condição humana.

A lo que agrega, profundizando la idea presentada, que:

É imprescindível distinguir a relação de poder e a relação de violência. Para ele, o poder é uma forma de exercer a dominação que se caracteriza pela legitimidade e pela capacidade de negociar o conflito e estabelecer o consenso, enquanto a violência é uma relação social inegociável, pois alcança, no limite, as condições de sobrevivência, materiais e simbólicas.

No famoso capítulo Superiority and subordination, de Simmel (1896), a dominação, assim como o conflito, não se encontra na imposição unilateral da vontade do subordinador sobre o subordinado, mas envolve uma ação recíproca. A ação do subordinador não pode ser compreendida sem a referência ao subordinado e viceversa, ou seja, ao contrário de violência, a dominação é uma forma de interação. ${ }^{7}$

En este contexto, la violencia siempre provocará un dolor, una herida, un daño; puede ser físico o psicológico; pero la aflicción estará presente en la experiencia de algún personaje de la historia narrada.

\section{La violencia hecha palabra}

Con este telón de fondo es fácil proponer la lectura de los cuentos de los tres autores hispanoamericanos elegidos, como el encuentro entre víctimas y victimarios; aunque definir quién es quién en estas narraciones -a veces- no es tan simple, ya que por la riqueza de los matices propuestos hay momentos en los que se confunde. Como rápido ejemplo señalamos a Julio Cortés, el personaje central de "Dolor profundo", del nicaragüense Juárez Polanco, quien va a intercalarse en esas funciones: en un instante es víctima, en el siguiente, victimario.

Hay quienes dirán que las relaciones de violencia siempre serán reflejo de la degradación de la humanidad. Sin abandonar totalmente la idea, en estos cuentos se muestra que también puede ser reflejo de una reacción -casi- natural (hasta con buenas intenciones se podría decir) del ser humano. Nuevamente utilizamos el cuento de Juárez Polanco para observar el silencio de la amante embarazada como la reacción "natural" y "amable" de parte de la violentada frente a la violencia -disfrazada de incomprensión y desconocimiento- ejercida por Julio Cortés.

El punto central está en que, en el universo planteado por los escritores que analizaremos, hasta

\footnotetext{
${ }^{6}$ ALVES de CARVALHO, Giane. Conflito, violência e tragédia da cultura moderna: reflexões à luz de Georg Simmel. Revista Brasileira de Segurança Pública. Ano 1, Edição 2, 2007, pág. 155.

${ }^{7}$ ALVES de CARVALHO, Giane. Conflito, violência e tragédia da cultura moderna: reflexões à luz de Georg Simmel. Revista Brasileira de Segurança Pública. Ano 1, Edição 2, 2007, pág. 156.
} 
algunos detalles amables se metamorfosearán para transformarse en pequeñas (o grandes) pesadillas. Quizás lo que profundice esa situación violenta sea justamente la involuntariedad de la acción. De todas maneras, de una u otra forma, el orden universal es roto, el otro es atropellado por la acción del violento y este produce dolor (o la muerte) en el (o del) victimario.

A veces creemos que la idea de violencia (culturalmente institucionalizada) corresponde exclusivamente a países (o sociedades) que la sufrieron de alguna manera extrema. Colombia, por ejemplo, tradicionalmente reconoce en sus estudios históricos - literarios un periodo conocido como "La Violencia", convencionalmente fechado entre 1948 y 1965, época de conflictos sociales y políticos, que dio como resultado artístico las "Novelas de la violencia".

No es el caso ni de Argentina ni de Perú, aunque las últimas dictaduras militares sirvieron como motivador de una larga serie de escritos que hablaron (y algunos todavía hablan) de la violencia institucionalizada por los gobiernos militares, de las víctimas de ese sistema, de los silencios obligatorios y las muertes innecesarias. En el caso específico de Argentina, la inverosímil guerra de Malvinas produjo una colección de novelas y cuentos que giraron sobre aquel vergonzoso conflicto, lo que -naturalmenteretoma la temática de una violencia institucionalizada.

El "problema" es que la violencia que los autores pintan en los textos que vamos a comentar en este artículo no pasa ni cerca de esos periodos. Es una violencia nueva, contemporánea, sin uniformes, sin militares, sin estructuras políticas ni narcotraficantes. Es una violencia instaurada en "la esquina de cualquier barrio"; "que puede pasar"; una violencia sin un motivo, porque cualquier motivo es suficiente. Una violencia que se viste como la ropa nuestra de cada día, que se alimenta con nuestro pan cotidiano. Una violencia que -muchas veces- nace, crece y muere en las relaciones más familiares.

En este sentido, las palabras de Velho ganan un profundo valor, cuando señala que: Mesmo com o final do regime militar fica patente que as relações com as camadas populares, apesar de trocas e interações, têm uma natureza cada vez mais explosiva. Há um enorme crescimento da criminalidade, com ampla divulgação pela mídia, tornando o cotidiano, especialmente nas grandes cidades, tenso e perigoso. Com o crescimento do tráfico de drogas associado ao de armas, montam-se redes criminosas com ramificações por todo o país, envolvendo contrabando, sequestros, que geram atmosfera de grande insegurança. A violência se dá em vários níveis, entre as próprias camadas populares, com grande número de mortos entre homens jovens, decorrentes também do confronto sistemático com a polícia e com os furtos, assaltos, sequestros e agressões de todos os tipos contra as camadas médias e superiores. ${ }^{8}$

Como observamos, es una violencia que se instaló en la sociedad, con razones más o menos complejas. Como el mismo Velho señala, esta realidad tiene relación directa con aspectos sociales que pasan por el gobierno (hipotéticamente) democrático de turno, la historia de cada grupo social y elementos que se fueron adueñando de espacios cada vez más representativos, como el narcotráfico.

A criminalidade e a insegurança nas ruas não chegam a ser uma absoluta novidade, mas nos últimos vinte anos têm atingido dimensões epidêmicas, afetando toda a sociedade. Já são feitas comparações com a violência na Colômbia e os índices, em geral, são alarmantes. A repressão policial, por sua vez, é extremamente violenta e constantemente indiscriminada em se tratando das camadas populares.

É preciso salientar que essa violência, ao lado de eventuais medidas filantrópicas do tráfico, é exercida, sobretudo, sobre as próprias camadas populares que ficam, assim, entre dois fogos em verdadeiras batalhas que são travadas nas favelas e na periferia das cidades. Algumas das gangues ou alguns de seus membros apresentam-se como protetores de suas "comunidades" contra a violência da polícia e atendendo as suas carências mais imediatas. $\mathrm{O}$ fato é que encontram apoios nessas localidades, confirmando,

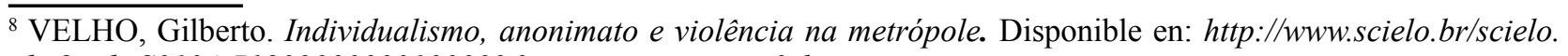
php? pid $=$ S0104-71832000000100002\&script $=$ sci_arttext\&tlng $=p t$ 
por exemplo, que laços de parentesco podem ter ainda importância, paralelamente às mudanças mencionadas. Sem querer simplificar uma complexa problemática, há que chamar atenção para o lado de performance da atuação desses jovens, para serem reconhecidos não só no seu meio, mas na sociedade maior, onde, através da mídia, atingem notoriedade, por mais efêmera e melancólica que nos possa parecer ${ }^{9}$

Estos jóvenes autores leen a los clásicos, leen a los maestros del siglo pasado, pero también leen blogs y páginas de Internet que son producidas por gente muy parecida a ellos; leen esos textos escritos por anónimos y por personas no especializadas que preguntan, comentan y observan la creación literaria desde una perspectiva diferente a la académica, que en muchos casos, no supera el básico y tradicional "me gusta" / "no me gusta".

En esta realidad, pese a la presión de las editoras para que se escriban (exclusivamente) novelas, los jóvenes autores continúan escribiendo (también) cuentos, narraciones breves que marcan nuevos senderos o que, dicho de otra manera, recorren los senderos antiguos pero proponiendo nuevas lecturas, quizás re-lecturas que señalan la característica constituyente -quizás básica- de esta nueva generación: la diversidad.

Como bien lo refleja el primer párrafo del artículo de Álvaro González, que aunque escrito en el año 2013 continúa actual, para El País Cultural (España) titulado La diversidad es la unidad en la actual literatura latinoamericana, cuando afirma:

La pluralidad de voces, la mezcla de razas y la hibridación de géneros son algunas características de la actual literatura de los 20 países que conforman América Latina. El retrato literario, si existiera, sería la diversidad y en el deseo de contar el mundo y contenerlo desde los micromundos que sus autores describen. ${ }^{10}$

Ritmos, vocabulario, paisajes, regiones... todo es diferente en cada escritor. Es difícil intentar encontrar algún elemento aglutinante en el universo propuesto por estas nuevas voces hispanoamericanas, entre las que señalamos -apenas para el análisis en este artículo- a la argentina Samanta Schweblin, al nicaragüense Ulises Juárez Polanco y al peruano Jeremías Gamboa. Si no es el único, al menos es uno de los pocos temas que pueden utilizarse como elemento de convergencia, es el asunto elegido para este artículo: la violencia. Cada uno de los escritores lo abordará de una manera diferente, distinta (lo que afirma la diversidad como característica fundamental de la época); pero todos ellos hablarán del asunto o lo mostrarán desde alguna de sus múltiples perspectivas.

Ellos tres, como tantos otros escritores nacidos en estas tierras latinoamericanas, nos presentan personajes que demuestran -en su accionar ficcional- que, por más que su narración parta de realidades extremamente concretas y específicas como pueden ser Buenos Aires, Managua o Lima, la violencia estará presente en el accionar, en las palabras, en el pensamiento de los habitantes de las capitales, de los pequeños pueblos y de las zonas rurales. Casi no hay fronteras para esta realidad que se ha instalado en nuestra sociedad.

\footnotetext{
${ }^{9}$ VELHO, Gilberto. Individualismo, anonimato e violência na metrópole. Disponible en: http://www.scielo.br/scielo. php? pid=S0104-71832000000100002\&script $=$ sci_arttext\&tlng=pt

${ }^{10}$ GONZÁLEZ, Álvaro. La diversidad es la unidad en la actual literatura latinoamericana. Disponible en: http://cultura. elpais.com/cultura/2013/12/07/actualidad/1386385815_918803.html Web. 27 de noviembre de 2015.
} 


\section{Más palabras. Más violencia.}

El cuento "Matar un perro" de Samanta Schweblin (Buenos Aires, 1978), publicado en su colección titulada El núcleo del disturbio ${ }^{11}$, es un excelente ejemplo de un texto producido con los parámetros de la literatura del siglo XXI y que muestra -con una claridad, para algunos, ejemplar- la nueva cadencia de las letras hispanoamericanas.

Con vocabulario simple, ritmo cinematográfico, descripciones precisas y relato directo, la escritora argentina presenta -resumidamente- la historia de un hombre (anónimo y amedrentado) que debe realizar una prueba de valor ( $¿$ ?) para poder ingresar en una organización criminal. En el caso: matar un perro.

El tema de la violencia domina el relato. Temática que se repite -de diversas maneras y desde distintos ángulos- en las otras páginas de sus colecciones de cuentos.

También será tema, por ejemplo, en el delirante relato del peruano Jeremías Gamboa (Lima, 1975) titulado "Un responso por el cine Colón" que forma parte de la colección Punto de fuga ${ }^{12}$, en el que la violencia se disuelve en risas -absolutamente impensadas en "Matar un perro"- pero que no consiguen esconder el momento de furia colectiva que se produce en aquel melancólico cine triple $\mathrm{X}$ del centro de Lima frente a la imagen de un Batman raquítico.

Es el mismo exceso, aunque pintado con tonos diferentes, que el nicaragüense Ulises Juárez Polanco (Managua, 1984) dibuja en el cuento "Dolor profundo", presente en el libro La felicidad nos dejó cicatrices $^{13}$ al hablar de un mundo de relaciones que desemboca en embarazos -reales y ficticios- que se descubren y se esconden al ritmo de posibles e imposibles abortos más o menos clandestinos.

No creo que sea un punto en común buscado por los autores (hasta porque la relación entre ellos no es tan fluida), pero la sociedad del siglo XXI nos coloca a nosotros -como ciudadanos-lectores- y a ellos -como ciudadanos-escritores- frente a una violencia constante que se verá reflejada en las obras de arte.

Como nos recuerda la escritora venezolana Carolina Jaimes Branger, en una entrevista para Informe 21 de su país, cuando dice:

Tenemos una sociedad deshumanizada por la violencia. Y eso no es la primera vez que pasa ni será la última desgraciadamente. Quizás el ejemplo más palpable y más cercano que tenemos es Alemania durante la guerra, cuando la gente se pregunta que por qué se murieron seis millones de judíos, que por qué no huyeron. No huyeron, sencillamente, porque se fueron acostumbrando a situaciones cada vez más catastróficas. Al principio era salir con una Estrella de David en la ropa, después era mudarse de sus casas para un gueto, después era salir del gueto al campo de concentración, cuando finalmente llegaron al campo de exterminio ya habían bajado la cabeza, de tan acostumbrados; nosotros nos hemos acostumbrado a la violencia. A nosotros ya no nos sorprende que maten a 150, 200 personas por fin de semana solamente en una ciudad de Venezuela, cuando tu llegas a un mercado y matan a una persona, está cubierta como con unas bolsas de basura porque la camioneta de la morgue no da abasto para buscar los cadáveres y la gente le pasa por encima, sin pensar que alguien ahí atrás puede ser un familiar. Es una deshumanización total. Y eso nos está pasando a todo nivel. ${ }^{14}$

La violencia descrita en estos cuentos es -lamentable y absolutamente- natural. No es una violencia institucionalizada, como la de las novelas de las dictaduras de los años 80. Tampoco es el reflejo literario del accionar de guerrillas tales como el M-19 o Sendero Luminoso. Es una violencia que nace en cualquier momento, en cualquier lugar, por cualquier circunstancia, casi sin motivo. Pero a la que nos vamos acostumbrando.

\footnotetext{
${ }^{11}$ SCHWEBLIN, Samanta. El núcleo del disturbio. Buenos Aires: Destino ediciones, 2002.

${ }^{12}$ GAMBOA, Jeremías. Punto de fuga. Madrid: Alfaguara, 2007.

${ }_{13}^{13}$ JUÁREZ POLANCO, Ulises. La felicidad nos dejó cicatrices. Granada: Valparaíso ediciones, 2014.

${ }^{14}$ JAIMES BRANGER, Carolina. Nos hemos acostumbrado a la violencia. Entrevista para Informe 21 (2014). Disponible en: https://www.youtube.com/watch? $v=j 3 l$ CitooK1M. Web. 17 de noviembre de 2015.
} 


\section{Como observa Cleia Schiavo Weyrauch ${ }^{15}$ :}

A violência urbana se manifesta de diversas formas, individual e/ou coletivamente, segundo a natureza do espaço público e/ou privado, da qualidade de seu processo de produção, urbanização e, sobretudo, do nível de privação de sua população no campo da sobrevivência e dos direitos sociais. As formas históricas de violência também variam segundo o design urbanístico do espaço, da qualidade do seu sistema sociopolítico cultural, do número de habitantes em um dado território e da consciência comunitária de seus habitantes. A cidade como lugar das desigualdades se intensifica em certos contextos históricos, a exemplo o da consolidação do capitalismo e suas consequentes transformações. Este quadro colabora na interiorização da violência na medida do grau de frustração com base nas aspirações e bloqueios advindos tanto do desejo de qualidade de vida quanto dos estímulos da sociedade de consumo dirigido. A violência urbana tornou-se um fenômeno sistêmico alimentado pela economia, pela política que expressa a dinâmica global da estrutura capitalista sobretudo em países como os da América Latina onde o nível de concentração de renda é espantoso.

\section{Relaciones violentas}

Quizás el ejemplo más contundente de la violencia instaurada en la sociedad sea el relatado en el cuento de la escritora argentina. En el mismo, los dos personajes centrales se construirán a través de sus palabras. Palabras breves, punzantes y directas que la narradora integra al texto sin avisarle a su lector.

"El Topo dice: nombre, y yo contesto" (p.177) ${ }^{16}$. Así comienza el cuento. Con esas palabras inicia la relación (breve, apenas un par de horas en una única noche que será suficiente para la decisión que debe ser tomada) entre dos hombres (El Topo y el anónimo narrador) que comparten un Peugeot que ahora conduce el personaje sin nombre que lo identifique.

La necesidad de dinero (y todas las fuerzas nefastas del capitalismo) hará que el personaje anónimo busque -en la noche- una opción (quizás) criminal para “solucionar su vida”. Schiavo Weyrauch comenta:

Na verdade, a violência estrutural do capitalismo aliou-se à da cultura da violência nas várias esferas do social, em uma cadeia perversa de resultados em que não se distinguem início e fim. No fundo, a violência da urbs expressa uma combinação de causas que elevam as frustrações da população ao nível da revolta. Trabalhadores vez por outra depredam trens, vans e ônibus, são homens e mulheres que, pressionados pelos estrangulamentos da cidade, vêem potencializados seus problemas específicos e reagem até criminalmente quando chegam ao seu limite ${ }^{17}$.

Observando el espacio nocturno que se elige para esta relación violenta, se hacen interesantes los detalles señalados por la mencionada autora ${ }^{18}$, cuando dice:

A violência faz a população recolher-se à noite ao interior das casas, enquanto uma população de rua, identificada como "criminosa", se instala sob as marquises e viadutos da cidade. A resistência desta população se revela na ocupação de prédios abandonados, na organização em movimentos.

"No me mira, dicen que nunca mira a nadie a los ojos. Edad, dice, cuarenta y dos, digo, y cuando dice que soy viejo pienso que él seguro tiene más" (p.177). La violencia no se da en la acción, sino en la

\footnotetext{
${ }^{15}$ SCHIAVO WEYRAUCH, Cleia. A violência urbana. Disponible en: file:///C:/Users/UCS/Downloads/2580-4000-1PB.pdf Web. 17 de noviembre de 2015.

${ }^{16}$ En este artículo, cada citación del cuento analizado será marcada apenas con el número de la página, pues siempre se utilizará la edición señalada en la bibliografía: El núcleo del disturbio. Buenos Aires, Destino ediciones, 2002.

${ }^{17}$ SCHIAVO WEYRAUCH, Cleia. A violência urbana. Disponible en: file:///C:/Users/UCS/Downloads/2580-4000-1PB.pdf Web. 17 de noviembre de 2015.

${ }^{18}$ SCHIAVO WEYRAUCH, Cleia. A violência urbana. Disponible en: file:///C:/Users/UCS/Downloads/2580-4000-1PB.pdf Web. 17 de noviembre de 2015.
} 
palabra, en el silencio, en la no-mirada. Los diálogos, extremadamente cortos y directos, generalmente reflejan preguntas muy específicas y respuestas escuetas, marcadas por brevísimos "dice" y "digo", que marcan el turno de habla de cada uno. Los comentarios del personaje anónimo siempre se quedarán en el nivel del pensamiento. No se harán palabra y mucho menos acción; aunque eso lo podría salvar del fracaso final.

Como se observa desde el mismo inicio del cuento, la violencia no necesita demostrarse en acciones, a veces una palabra construye una realidad simbólicamente más violenta que una lucha corporal.

En estos últimos años no se puede hablar de violencia simbólica sin remitirnos al francés Pierre Bourdieu $^{19}$, ya que sus ideas permean nuestro actual pensamiento sobre este asunto por considerarla como un instrumento al servicio no apenas de la clase dominante, sino de todos los agentes sociales; con la diferencia que -en el caso del relato que estamos analizando- el "dominante" presiona al otro, mientras que el "dominado" apenas consigue pensar en un accionar violento.

Está justamente en este elemento la mayor diferencia entre los dos. El Topo, el dominante, se construye a través de la palabra dicha. El otro, el dominado, a pesar de utilizar la palabra (¿o se la niega -¿por miedo?- a sí mismo?), no consigue construirse, no consigue ser. No es ni será asesino, ni matador, ni ejecutor: ni de perros ni de hombres ni de pensamientos.

Jeremías Gamboa en su "Un responso por el cine Colón”" también aborda, desde una óptica diametralmente opuesta, el mismo tema; en este caso, diríamos apenas una violencia. Como en el caso de la narradora argentina, los hombres (en el caso específico del narrador peruano, sexo masculino exclusivamente) se crean como personajes por sus palabras. Será el elemento que los una para protestar contra la peor película XXX -según la valoración del personaje central del cuento- alguna vez exhibida en aquel cine del centro de Lima.

Será también el lenguaje el elemento que el escritor le ofrecerá a su personaje -desesperado para publicar algún artículo en alguna revista- para que intente ser lo que -realmente- no es. No en vano, el personaje que relata su experiencia frustrante en aquella sesión cinematográfica inolvidable, negativamente inolvidable, todo lo que tiene para ofrecer son palabras, historias con mucho de extraño, algunas que rozan lo ridículo; pero la elegida como eje central para su gran intento de convencimiento, es el relato de un momento de violencia compartida por los integrantes -desconocidos y anónimos- de una red de relaciones circunstancial y momentánea.

Los hombres que están compartiendo la sesión de una película pornográfica en un viejo y decadente cine-teatro del centro de Lima se unen en una protesta (verbal al inicio, física después) contra la película que están mirando.

La historia, en realidad, comienza en otra circunstancia, en otro momento y en otro lugar. Felipe Castrejón -una vez más- le intenta vender un reportaje, para cobrar lo que sea, a su amigo - editor. El tema es sobre un concurso de feos. En el periodo de la espera laboral obligada (el diseñador de la revista tiene que realizar su trabajo) aparece la salida de la redacción, el pisco compartido y la verdadera historia del cuento. Esta inicia en la adolescencia del personaje, con sus recorridos vespertinos por los diferentes cines XXX de los distintos barrios de Lima. Llega a su momento culminante cuando Castrejón consiga llegar -con menos vergüenza y un poco más de edad- a la "sórdida letrina de cine pornográfico a la que solo acudían tipos arrechos y sin plata para comprarse un VHS” (p.84), según las palabras del narrador.

La respuesta -más que respuesta, defensa- del intento de periodista, corre por la arquitectura del

\footnotetext{
${ }^{19}$ BOURDIEU, Pierre. O poder simbólico. $4^{\mathrm{a}}$ Ed. Rio de Janeiro: Betrand Brasil, 2001.

${ }^{20}$ En este artículo, cada citación del cuento analizado será marcada apenas con el número de la página, pues siempre se utilizará la edición señalada en la bibliografía: Punto de fuga. Buenos Aires, Alfaguara, 2007.
} 
espacio, la historia de la edificación y por la importancia que el local tuvo en su vida: "Desde entonces no fui a ningún otro sitio -me dijo Castrejón dándole luego un sorbo a su pisco sour-. En el Colón me quedé para siempre, te juro" (p.87).

La pregunta central del relato la plantea el narrador: ¿Qué pasó en aquel cine una tarde cualquiera? La respuesta: "Una revolución -me dijo, entonces-, una verdadera revolución. Y derrocamos a Batman" (p.90).

Más allá de lo absurdo de la situación planteada, el concepto de revolución ya se acerca a la idea de violencia. Por el contexto en el que se presenta, es difícil tomarla en serio. Parece más una burla a la violencia; pero si bien no se van a encontrar a personajes como El Topo, de Samanta Schweblin, las expresiones furiosas comienzan en el final de la primera película de la serie propuesta para esa tarde: silbidos, chiflidos y rechiflas generalizadas. En ese proceso, in crescendo, Castrejón se une al grupo revolucionario, a los silbidos y al rechazo general de lo que la película está mostrando.

Llega la arenga final, que

atronó en el lugar como una descarga eléctrica o el grito de guerra que antecede a la hecatombe [...] Como si hubieran identificado al enemigo, los hombres empezaron a pararse de sus asientos y a lanzar a voz en cuello una sarta de improperios contra el actor, la película, los administradores del teatro, los dueños y, por supuesto, contra las madres de estos (p. 96).

En esa locura compartida:

de pronto todos estábamos parados delante de nuestros asientos y solo unos segundos después un hombre se salió de las butacas, subió las escaleras del escenario y se puso a golpear el ecran, a batir la tela y luego otros más se subieron también sobre sus asientos [...] algunos habían trepado hacia la cabina de proyección (p. 97).

En este caso, se consigue el objetivo: "la película paró" (p. 97).

Julio Cortés, el personaje central ya mencionado del cuento "Dolor profundo" ${ }^{21}$ queda helado en una las callejuelas del laberíntico Mercado Oriental de Managua, cuando una niña lo identifica como el futuro cliente del Señor de las cunas.

Pensando iba, cuando una niña le salió al paso:

- ¿Qué anda buscando, señor? ¿Seguro que no quiere que le venda algo? Quizá para su muchacha...

- Nada, nada. Ando buscando unas cosas para la casa. Dame lugar...

- Tengo unas cunas bien bonitas para usté. (p. 108)

La violencia está en la palabra, no porque sea dura o soez, sino porque revela, descubre, desnuda a la persona y sus intenciones.

El asunto es el siguiente. Como te tardaste en actuar, hay que entrar y sacar. Eso lo hago yo, rápido, en unos minutos. Después, yo desaparezco, me hago humo, pero te ofrezco asesoría y acompañamiento a distancia después del procedimiento. Soy médico, o lo fui, es lo mismo. Todo esto es $100 \%$ seguro, pero por si acaso, en caso de irregularidad posterior, llamás a este número, avisás, y te me vas al Vélez Páiz, ahí vas a emergencias, preguntás por Carlita o Martita que ellas te atienden sin hacer preguntas. Por todo este servicio, son mil verdes. ¿Hay trato? (p. 109)

\footnotetext{
${ }^{21}$ En este artículo, cada citación del cuento analizado será marcada apenas con el número de la página, pues siempre se utilizará la edición señalada en la bibliografía: La felicidad nos dejó cicatrices. Granada, Valparaíso ediciones, 2014.
} 
La violencia descrita en la escena del primer encuentro entre el novio que no quiere ser padre y el hombre que se propone a realizar el aborto clandestino. El pacto se sella con un abrazo; "el abrazo de un caníbal listo a devorar su almuerzo" (p. 110).

Es una violencia más simbólica, que se consigue esconder entre conversaciones que no dicen nada en concreto y sospechas que -el lector- va dejando crecer a medida que las informaciones van ocupando las líneas del cuento. Por el momento, quien es violento para con su futuro hijo, se siente violentado por quien realizará el aborto. La novia, a quien intenta -de alguna manera- proteger, no le responde sus llamadas. Para completar el cuadro, el Toyota Yaris del joven administrador se estaciona delante de un apartamento de la Colonia Centroamérica, uno de los barrios populares de la ciudad, "a las cinco de la tarde" (p. 111), donde vive Luisa Ventura, su amante, quien acepta su papel en silencio, sin informar -siquiera- su condición de embarazada. Una historia urbana. ¿Una muestra de violencia urbana?

Más allá de la descripción de la violencia que cualquiera de los textos presente, lo que es simple observar es el proceso por el cual uno domina (económica, social o culturalmente) al otro y cómo este (el dominado) acepta esa situación como siendo natural. Es el silencio de Julio Cortés frente al Señor de las cunas, y de Luisa Ventura frente a Julio Cortés; el silencio del anónimo narrador frente al Topo y del responsable técnico del cine frente al accionar de la patota de hombres indignados por las escenas proyectadas. Es el reflejo de una sociedad en la que la violenta ganó un espacio que no quiere abandonar.

El sistema simbólico de una determinada cultura es una construcción social, generalmente larga y compleja en su proceso, que se mantiene en el tiempo gracias a la fundamental perpetuación que los agentes sociales realizan -consciente o inconscientemente- del mismo. De esta manera, la violencia simbólica se expresa en la imposición legítima (para ambos grupos, los dominantes y los dominados) y en la interiorización de ese sistema en los agentes de la cultura. El dominado no se opone a su opresor porque no consigue percibirse como víctima; por el contrario, considera que esa es la situación natural e inevitable.

Con este sombrío telón de fondo, las relaciones planteadas en los textos, en tres contextos diferentes, con matices y elementos diferenciadores, se pueden unir en este concepto básico: las diversas relaciones violentas marcan la cultura latinoamericana que se refleja en los cuentos que estamos comentando.

\section{Cualquier espacio es lo mismo para la violencia ( $₫$ ?)}

Como hemos visto, cada uno de los cuentos aborda la temática desde una óptica diferente. Pinta esta misma realidad social con diversos colores, algunos más trágicos, otros, menos. Mientras Samanta Schweblin hace pasear al pobre ser anónimo y acobardado por las calles de Buenos Aires para que (no) cumpla su misión -matar un perro-, Jeremías Gamboa apagará las luces del cine-teatro Colón para encenderlas nuevamente y desnudar los rostros de los habitués del lugar y la arquitectura de la sala, y Ulises Juárez Polanco llevará a la pareja de (casi) tres hasta el lugar donde se practicará el aborto clandestino, que no será necesario practicar porque la novia (oficial) no está embarazada, aunque la amante sí lo esté y el novio - amante no lo sepa.

Si bien cada una de las situaciones planteadas tiene un espacio determinado, no sería absurdo pensar en un cambio de lugar sin modificar -para nada- las historias narradas. Es natural que cada narrador haya elegido ubicar su relato en su espacio vital (Samanta Schweblin en Buenos Aires, Jeremías Gamboa en Lima y Ulises Juárez Polanco en Mangua), pero podríamos encontrar a un personaje anónimo con un 
Topo en Lima o en Mangua, al mismo tiempo en que se podría descubrir a Julio Cortés en Buenos Aires o en Lima. Esas opciones de mudanza marcan una posibilidad de relacionarse desde cualquier espacio con el mundo, con ese hábitat general que se transforma en el hogar de todos, pues las relaciones de violencia planteadas se las puede ubicar -sin mayores dificultades- en cualquiera de los espacios latinoamericanos que fueron señalados.

Como una suerte de "Pierre Menard, autor del Quijote"22 espacial, podemos utilizar la propuesta final borgeana de aquel cuento (que es presentada desde la óptica temporal) para observar esa movilidad cultural que la sociedad latinoamericana de hoy ofrece.

Parece que en la actualidad hay una violenta homogeneidad social en los espacios de las ciudades latinoamericanas que cualquiera de ellas podría ser propicia para transformarse en el espacio ficcional vital de cualquiera de los personajes presentados en cualquiera de los cuentos que estamos comentando. Quizás la pregunta sea: ¿cualquier ciudad del mundo? La respuesta ocuparía las páginas de otro estudio.

Lo que sí queda claro es que la violencia no conoce fronteras, se repite en su esencia destructora en todos los espacios y nuestra América Latina la sufre oficial, social y simbólicamente. La literatura la refleja en todas sus dimensiones.

Recebido em: 01/03/2016. Aprovado em: 02/06/2016.

${ }^{22}$ BORGES, Jorge Luis. Cuentos completos. Buenos Aires: Sudamericana, 2012, pág. 91-98. 